\title{
THE MICROSTRUCTURE AND MECHANICAL PROPERTIES OF TITANIUM DIOXIDE NANOTUBES SYNTHESIZED IN THE FLUORIDE-BASED ELECTROLYTE
}

\author{
Ying Pio Lim ${ }^{1 *}$, Wei Hong Yeo \\ ${ }^{1}$ Faculty of Engineering and Quantity Surveying, \\ Inti International University, Malaysia. \\ ${ }^{2}$ Lee Kong Chian Faculty of Engineering and Science, \\ Universiti Tunku Abdul Rahman, Malaysia.
}

Received 07.12.2017

Accepted 02.07.2018

\begin{abstract}
Titanium is one of the biomaterials commonly used for prosthetic devices due to its bio-inert properties. The discovery of titanium dioxide nanotubes (TDNTs) has created a great interest in medical applications such as dental and orthopedic implants. The synthesizing of TDNTs can produce different morphology, sizes and mechanical properties of the nanotubes - depending on the applied method. In this study, an electrochemical anodization method was used for synthesizing the TDNTs. A $100 \mathrm{ml}$ mixture of $99 \%$ of ethylene glycol (EG), $1 \%$ of deionized water and 1 wt.\% of ammonium fluoride $\left(\mathrm{NH}_{4} \mathrm{~F}\right)$ was used as the electrolyte of the electrochemical cell. Parameters such as anodization time and the voltage applied were used to alter the morphology of the TDNTs formed. The produced nanotubes were analyzed and characterized using scanning electron microscopy (SEM), X-ray diffraction (XRD) and microhardness tester. The SEM results showed that the formed diameter of nanotubes was mainly affected by the anodizing voltage. The wall thickness was found to be irrelevant to the parameters conducted in this study. The diameter of nanotubes formed with an anodizing voltage of 30, 45 and $60 \mathrm{~V}$ have the diameters ranging from $46 \mathrm{~nm}$ to $71 \mathrm{~nm}$. All of the TDNTs samples formed have a wall thickness between $11 \mathrm{~nm}$ and $13 \mathrm{~nm}$. With the use of EG and $\mathrm{NH}_{4} \mathrm{~F}$ as an electrolyte, the array of TDNTs with honeycomb structure was formed. In general, hardness test showed that the hardness of the nanotubes was inversely proportional with the anodizing time. The anodizing voltage only has little effect on the hardness of the nanotubes. The nanotubes formed by $60 \mathrm{~V}$ have about 3 to $5 \%$ lower hardness compared to those formed by $30 \mathrm{~V}$ for different anodizing times.
\end{abstract}

\footnotetext{
*Corresponding author: Ying Pio Lim, yingpio.lim@newinti.edu.my
} 
Keywords: Synthesis, titanium dioxide nanotubes; electrochemical anodization method; dental and orthopedic implants.

\section{Introduction}

Titanium is a metal commonly used as a biomaterial in the prosthesis and biomedical devices. The oxide layer on titanium surface protects the titanium matrix from corrosion, and it is biocompatible. Due to these facts titanium has become a metal that is widely used in dental and orthopedic implants [1].

With the discovery of titanium dioxide nanotubes (TDNTs) in 1998 by Kasuga and co-workers [2], the application of TDNTs as bone and dental implants have become an interest due to the similarity of the surface morphology between the human bones and TDNTs. The TDNTs can help create a larger surface area to ensure good osseointegration. Other than that, the nanotubes can also serve as reservoirs for drug delivery system to prevent infection. Various routes including sol-gel, template-assisted, hydro/solvothermal approaches, and by electrochemical means (anodization) may achieve a synthesis of $1 \mathrm{D} \mathrm{TiO}_{2}$ nanotubes. Among these methods, anodization is a relatively more straightforward and economically feasible method.

The anodizing conditions are essential to alter the characteristic of nanotubes formed during the synthesis process. The length of the nanotubes formed is longer when using the organic solution compared to the aqueous solution as electrolytes [3]. Besides that, a more acidic electrolyte can form shorter nanotubes [4]. Another factor that could affect the length of nanotubes is the anodizing time where longer anodizing time produced longer nanotubes [2].

The concentration of fluoride ions in the electrolyte and the anodizing voltage applied can change the diameter of the TDNTs produced. The study done by Zwilling et al. [5] concluded that a small addition of fluoride ions is the key to form the TDNTs using anodization. A progressive increase in fluoride concentration can reduce the diameter of the nanotubes [6]. On the other hand, increasing the anodizing voltage increases the nanotubes diameters [7].

Recently many studies have reported antibacterial properties of $\mathrm{Cu}$ material coated titanium alloys [8]. The presence of $\mathrm{Cu}$ element improves the mechanical properties, bio-corrosion and antibacterial properties of $\mathrm{Ti}-\mathrm{Cu}$ alloys for biomedical application [9]. As copper is one of the promising metal dopants for alloys in biomedical application, this research uses $\mathrm{Cu}$ as the cathode for the formation of TDNT.

\section{Objectives}

In real life situation, different types of prosthesis applied at different parts of the human body require specific physical characteristic especially the surface morphology and the hardness. Surface morphology is essential regarding bonding between the human bone cell and the coating itself. Appropriate surface morphology will enhance the cell-adhesion and hence improve the rate of recovering. As for the hardness, rigid contact may be provided at the implant area to have a sustainable design. This study investigates how the anodizing parameters (anodization time and anodization voltage) affect the surface morphology and hardness of TNDTs. Hence, suitable parameters can be ascertained to suit the need of the prosthesis. 


\section{Experiment}

The materials used in these experiments are ethylene glycol (EG), ammonium fluoride $\left(\mathrm{NH}_{4} \mathrm{~F}\right)$, titanium foil, copper foil, ethanol $\left(\mathrm{C}_{2} \mathrm{H}_{5} \mathrm{OH}\right)$, acetone $\left(\mathrm{C}_{3} \mathrm{H}_{6} \mathrm{O}\right)$ and deionized water. The reagent grade ethylene glycol with $99.5 \%$ purity and reagent grade ammonium fluoride with $98 \%$ purity are purchased from Friendemann Schmidt Chemical. The copper foil ( $99.9 \%$ trace metals) with a thickness of $0.1 \mathrm{~mm}$ and titanium foil $(99.7 \%$ trace metals) with a thickness of $0.127 \mathrm{~mm}$ are purchased from SigmaAldrich Chemistry.

The titanium foils used for anodization were ground with different SiC emery papers and followed by polishing with $6 \mu \mathrm{m}$ and $1 \mu \mathrm{m}$ diamond paste to produce an optically reflective surface. Then it was immersed in acetone and sonicated in an ultrasonicator for 15 minutes. Subsequently, the titanium foil was washed and immersed in deionized water followed by 15 minutes of sonication. After that, the titanium foil was immersed in ethanol and sonicated for 15 minutes. Lastly, the titanium foil was washed and dried in the ambient room.

In the setup of the electrochemical cell, the titanium foil was connected to the anode of a DC power supply with a clip wire whereas the copper foil was connected to the cathode of the DC power supply with a wire. Copper foil was used in cathode because it is cheap and has been reported to be able to exhibit good biocompatibility for biomedical application $[10,18]$. Both the foils were then immersed in a beaker containing $100 \mathrm{ml}$ of electrolyte. The electrolyte is made up of $99 \%$ of ethylene glycol, $1 \%$ of deionized water and $1 \mathrm{wt} \%$ of ammonium fluoride. A magnetic stirrer was used to stir the electrolyte continuously throughout the anodizing process.

Once the electrochemical cell was set up entirely, the titanium and copper foil was connected to DC power supply with $30 \mathrm{~V}$. The anodization time was set for 1 hour, 3 hours and 5 hours respectively for different runs. The experiment was repeated by changing the applied voltage to $45 \mathrm{~V}$ and $60 \mathrm{~V}$ with an anodizing time of 1 hour, 3 hours and 5 hours respectively. All anodized samples were characterized by scanning electron microscopy (SEM), $\mathrm{x}$-ray diffractometer and microhardness tester.

\section{Results and Discussion}

\section{Microstructural analysis}

The $\mathrm{TiO}_{2}$ nanotubes formed were viewed by using the high-resolution scanning electron microscope (HRSEM). The SEM micrographs for each voltage are shown in Fig. 1 to Fig. 9 for the anodizing times of 1 to 5 hours. The dimensions of wall thickness and tube diameter were measured by software's tool when the desired micrographs were captured. 


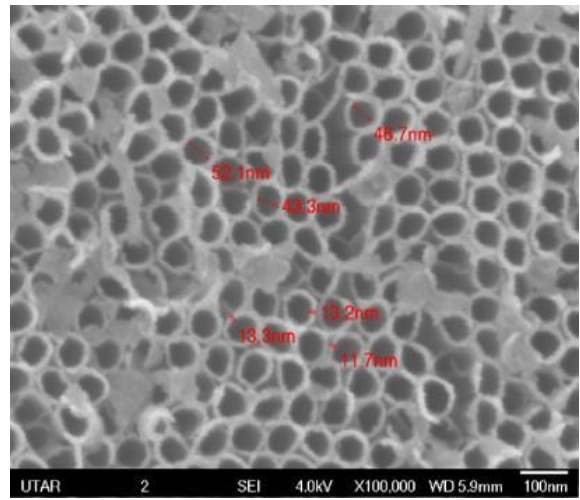

Fig. $1.30 \mathrm{~V}$ and 1 -hour anodizing time.

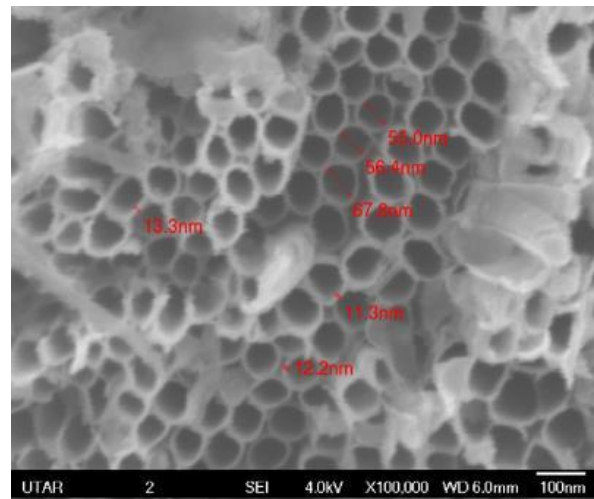

Fig. 3. $30 \mathrm{~V}$ and 5 hours anodizing time.

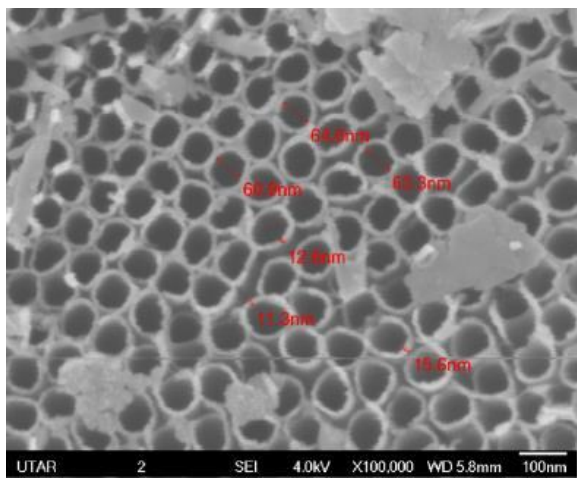

Fig. 5. $45 \mathrm{~V}$ and 3 hours anodizing time.

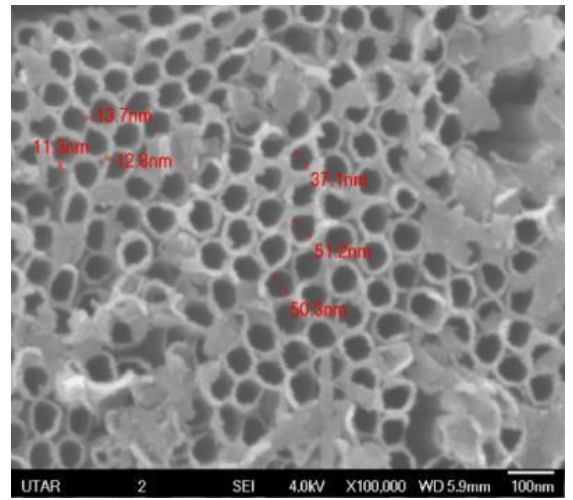

Fig. 2. $30 \mathrm{~V}$ and 3 hours anodizing time.

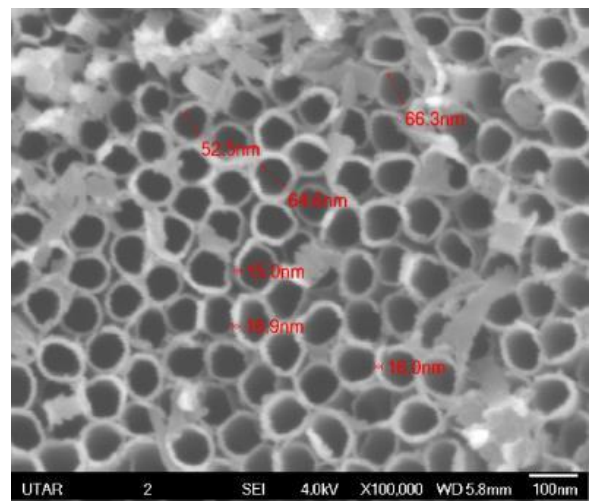

Fig. 4. $45 \mathrm{~V}$ and 1-hour anodizing time.

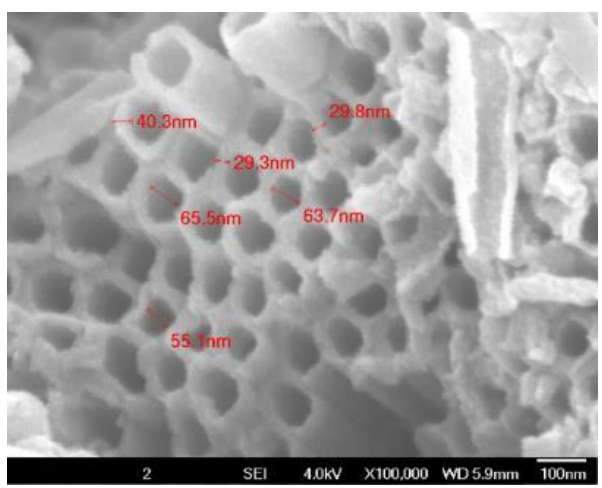

Fig. 6. $45 \mathrm{~V}$ and 5 hours anodizing time. 


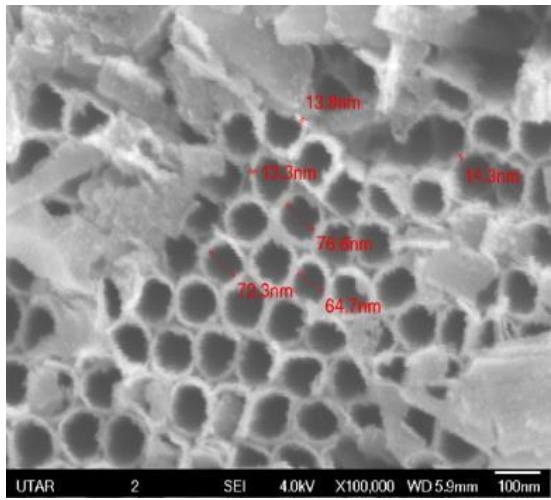

Fig. 7. $60 \mathrm{~V}$ and 1-hour anodizing time.

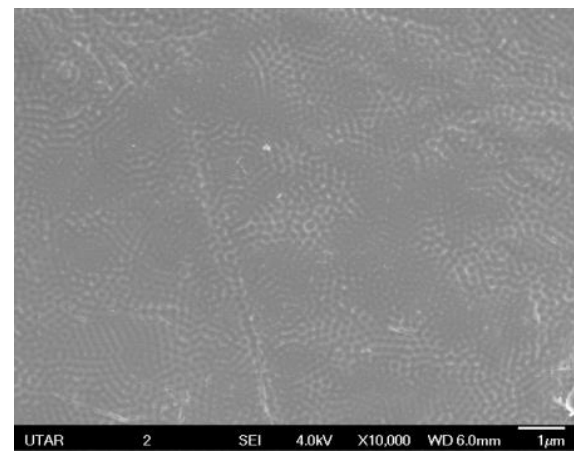

Fig. 9. $60 \mathrm{~V}$ and 5 hours anodizing time.

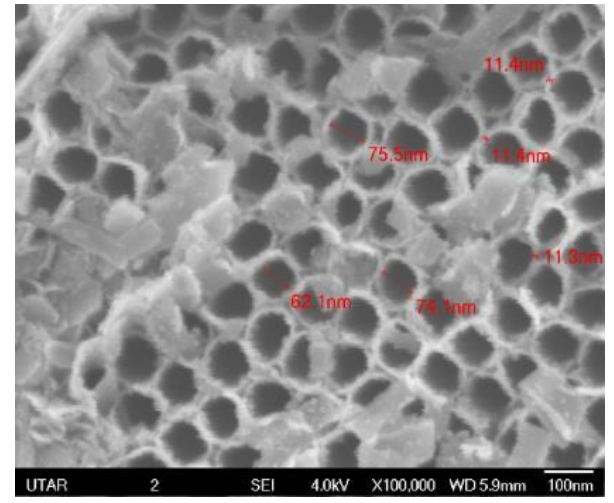

Fig. 8. $60 \mathrm{~V}$ and 3 hours anodizing time.

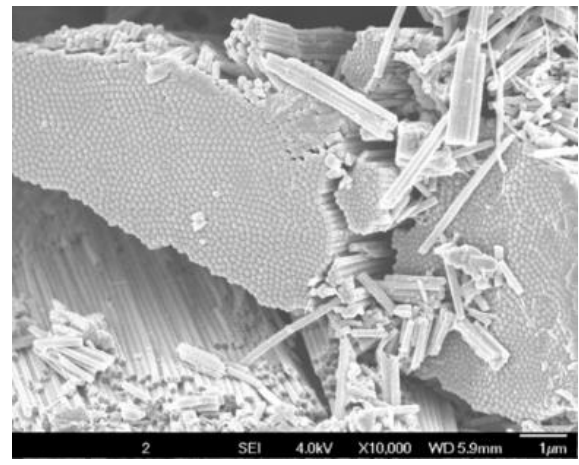

Fig. 10. Broken pieces of nanotubes formed under $60 \mathrm{~V}$ with 5 hours of anodizing time.

The nanotubes are a well-aligned 1D structure in a specific growth direction (Fig. 1-10). The nanotubes formed under $45 \mathrm{~V}$ have thicker wall compared to those samples synthesized under a voltage of $30 \mathrm{~V}$. Besides that, the nanotubes wall thickness is increasing proportionally to voltage. This observation is in line with the finding in Bauer et al. [7] whereby the authors found that the wall thickness of nanotubes linearly depends on the applied voltage. Also, the wall thickness of the nanotubes in the sample anodized with $45 \mathrm{~V}$ for 5 hours has increased by $160 \%$ compared to those anodized for 3 hours. This phenomenon could be due to the longer anodizing time at a higher voltage which leads to the extensive formation of the $\mathrm{TiO}_{2}$ coating on the nanotube's wall. This finding is inline the with the observation reported in Bauer et al. [7], whereby the authors found that the wall thickness of nanotubes linearly depended on the applied voltage [11].

For the nanotube arrays formed with $60 \mathrm{~V}$ of applied voltage, the surface structures were more irregular and rough. The well-aligned structure can only be obtained in some small areas. As for the sample of $60 \mathrm{~V}$ with 5 hours of anodizing time, the nanotubes were all covered up. The self-organized alignment of the nanotubes is still present even if there are many broken pieces of the nanotubes on the coating (Fig. 10). 
A similar observation was reported by Qingyun et al. [12]; they found that the appearance of tubes was lost and discrete, hollow, cylindrical tube-like features was created. At the voltage higher than $40 \mathrm{~V}$, the structure of nanotube is lost. This losing of nanotube structure could be attributed to over dissolution of tubes by fluoride ions [13]. Hence, the formation of the self-organized $\mathrm{TiO}_{2}$ nanotubes coating is less effective under high voltage [14].

Calculating the average diameter of the nanotubes in each sample allows studying the effects of experimental parameters on the formed $\mathrm{TiO}_{2}$ nanotubes morphology (Fig. $11)$.

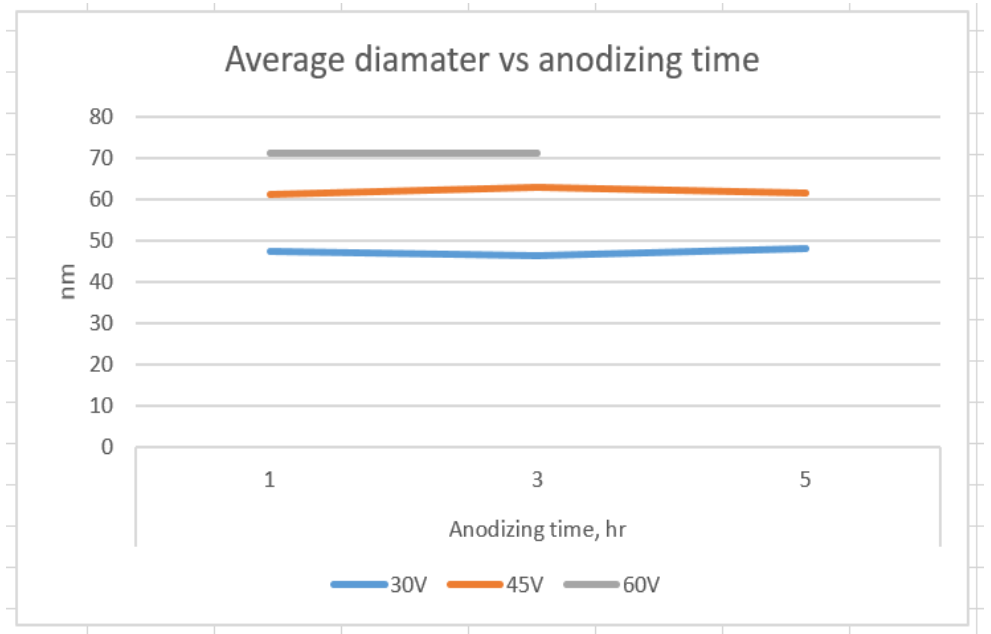

Fig. 11. Graph of average diameter vs. anodization time at different voltages.

The average diameter of the $\mathrm{TiO}_{2}$ nanotubes increases gradually from 30, 45 to $60 \mathrm{~V}$. At $30 \mathrm{~V}$ of the applied voltage, the average diameter of the nanotubes is from 46 to $48 \mathrm{~nm}$ for all anodizing times. For 45 and $60 \mathrm{~V}$, the average diameter is 61 and 71 $\mathrm{nm}$, respectively. In general, it is evident that the anodization time does not significantly affect the diameter of $\mathrm{TiO}_{2}$ nanotubes [15].

Fig. 12 shows the average wall thickness as a function of anodizing time at different voltages. The wall thickness of the $\mathrm{TiO}_{2}$ nanotubes formed was not significantly affected by the anodization time of 3 hours and below. The average wall thickness of all the samples ranges from 12 to $16 \mathrm{~nm}$. Nevertheless, the sample under 45 $\mathrm{V}$ with 5 Hours of anodizing time was found to insistent with the trend; this could due to the result of the instability of the anodizing condition. 


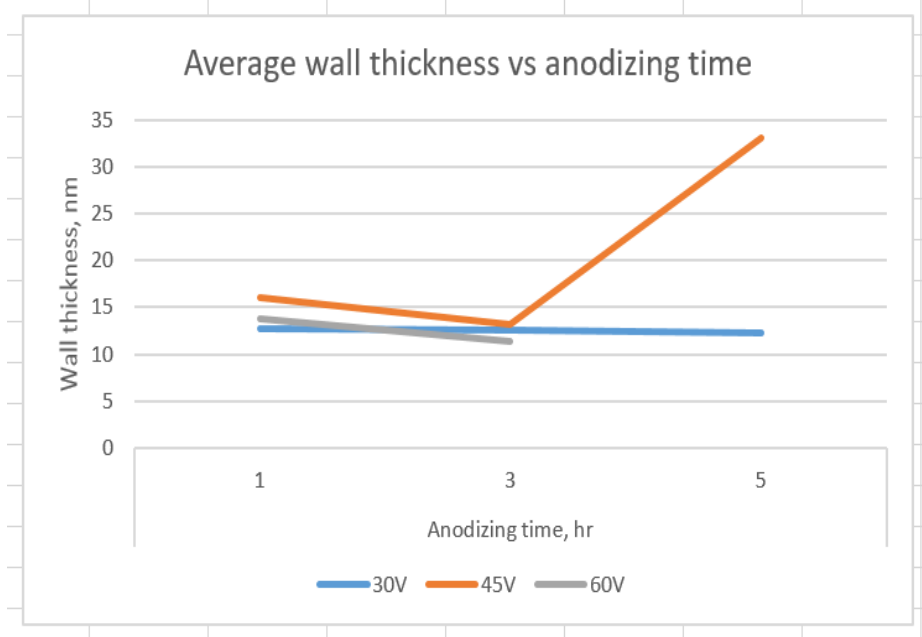

Fig. 12. Graph of average wall thickness vs. anodization time at different voltages.

The data indicates that the optimal anodizing time for thin wall thickness is 3 hours and below. The average wall thickness of all the samples, for these times, is around 12 to $16 \mathrm{~nm}$. Nevertheless, there is an alien data which is the sample under $45 \mathrm{~V}$ with 5 hours of anodizing time. The data indicates that the optimal anodizing time for thin wall thickness is 3 hours and below.

\section{$X$-Ray Diffraction (XRD) Analysis}

The samples are investigated using the X-Ray Diffraction (XRD), to determine the composition of the anodized titanium foil. Firstly, the significant peaks can be obtained by observing the $\mathrm{x}$-ray card data of titanium.

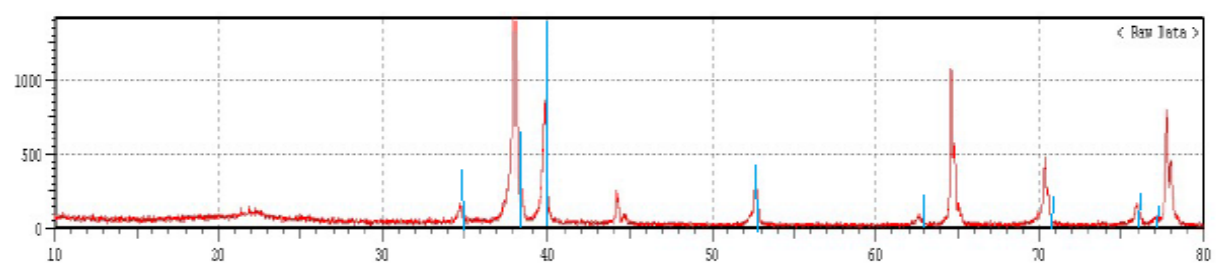

Fig 13. The X-ray card data of titanium (blue line), and XRD result of the original titanium foil (red line).

As shown in the Fig. 13, the three most significant peaks of the titanium are approximately located at $35^{\circ}, 38.5^{\circ}$, and also $40^{\circ}$ [16]. Next, the peaks of the X-ray card data are used to compare with the XRD result of the original titanium foil.

By comparing all the peaks of both spectra in Fig. 13, we can observe that the intensity of the peaks is different. However, the location of the peaks is similar to each other as the three most significant peaks of original titanium foil fall at about $38^{\circ}, 40^{\circ}$ and also $65^{\circ}$ to indicate the presence of Ti. XRD tests were carried out on all samples to compare the peaks with that of the original titanium foil [17]. 
By comparing the XRD results of the nanotubes formed under $30 \mathrm{~V}$, the three most significant peaks are all located at the same degrees which are about $38^{\circ}, 40^{\circ}$ and also $70^{\circ}$. However, the intensity decreases as the anodization time increases from 1 hour to 3 hours, and increases again at the 5 hours of anodization time. The decreasing of the intensity of titanium indicates that the composition of the titanium is gradually decreasing.

For the XRD results of the nanotubes formed under $40 \mathrm{~V}$ of applied voltage, the trend of intensity is similar to the samples of $30 \mathrm{~V}$. The intensity decreases at first from 1 to 3 hours and grows back at 5 hours. Nevertheless, the location of the three most significant peaks is matching with the XRD result of the original titanium foil which is $38^{\circ}, 40^{\circ}$ and $70^{\circ}$.

As for the samples of nanotubes formed under $60 \mathrm{~V}$ of applied voltage, the intensity decreases as the anodization time increases. The location of 3 most significant peaks is about $38^{\circ}, 40^{\circ}$ and also $71^{\circ}$. However, the last sample shows a very inconsistent result with more significant peaks compares to the other samples. This could be due to the failure in forming the nanotubes structure which in turn affects the overall composition of the sample.

As a conclusion, the formation of $\mathrm{TiO}_{2}$ nanotubes is not efficient for an extended period of anodizing time. This can be proven by the results of the XRD which show inconsistency at 5 hours of anodizing time. This situation is similar to the HRSEM test which shows irregularity of average diameter and also walls thickness at the 5 hours of anodizing time.

\section{Micro Hardness Test}

The setting of the microhardness tester is set to $0.5 \mathrm{~kg}$ of force with a dwell time of 10 seconds. With the aim of getting results with higher accuracy, the hardness of each $\mathrm{TiO}_{2}$ nanotubes sample is measured at three different locations on the surface. The average Vickers hardness value (HV) will then be calculated. The average hardness values of samples are plotted in Fig. 14 below.

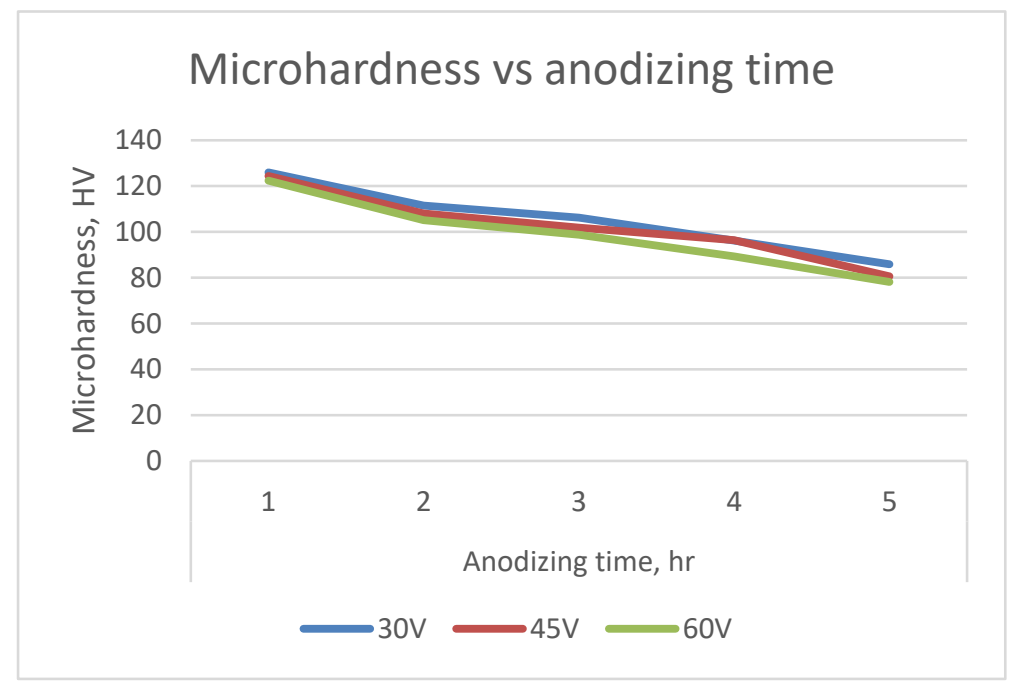

Fig. 14. Graph of average hardness vs anodizing time at different voltages. 
From the Fig. 14, it is observed that the average hardness value decreases as the anodizing time increases. According to the research by Haring, Morris and Hu [19], the diameter of the $\mathrm{TiO}_{2}$ nanotubes increases as the voltage applied to the anodization increases, which is verified in our results. The strength of the nanotubes with larger diameter was reported to be weaker compared to the strength of the nanotubes with a smaller diameter, assuming the wall thickness remains constant [19]. Also, during high anodizing voltage, the rate of movement of ions towards the electrode increases. Eventually, the potential difference between cathode and anode slowly increases which will result in higher rate of anodization. Consequently, the hardness of the nanotubes decreases when the rate of nanotubes formation increases.

\section{Conclusions}

The objective of this study is achieved successfully by forming the $\mathrm{TiO}_{2}$ nanotubes at different parameters of anodization time, and the applied voltage by using the electrochemical anodization method.

The micrographs of the formed nanotubes can are observed under the highresolution scanning electron microscope (HRSEM). The self-organized structure of the $\mathrm{TiO}_{2}$ nanotubes coating can be seen. This proves that the self-organized $\mathrm{TiO}_{2}$ nanotubes can be formed via the electrolyte with the mixture of ethylene glycol, deionized water, and ammonium fluoride. Next, the average diameter of the $\mathrm{TiO}_{2}$ nanotubes of each sample is calculated. The average diameter does not increase with the anodization time, but higher anodizing voltage will increase the average diameter. At the same time, the average wall thickness of the samples is also calculated, and the result shows that wall thickness is affected by both the parameters. Nonetheless, there is some inconsistency of result in the samples with a more prolonged period of anodizing time. The formation of $\mathrm{TiO}_{2}$ nanotubes coating is not suitable for the more prolonged period of anodizing time and also at a voltage higher than $30 \mathrm{~V}$.

As for the X-ray diffraction analysis, the three most significant peaks of each sample are successfully matching with the XRD result of original titanium foil. This shows that the composition of the titanium foil after the anodization process remains constant. Moreover, the intensity of the peaks decreases as the anodization time increases which indicates that the composition of titanium decreases. Lastly, the microhardness test of the samples gives consistent results. The hardness value of the samples decreases as the anodization time and the voltage applied increase. This could be associated with the higher forming rate of $\mathrm{TiO}_{2}$ nanotubes under a higher voltage applied.

\section{References}

[1] A. Robin, A. Ribeiro, J. L. Rosa, R. Z. Nakazato, M. B. Silva: J Surf Eng Mater Adv Technol, 4 (2014) 123-130.

[2] D. Porton, K. Papaefthymiou, C. Pirvu, G. Papanicolaou, I. Demetrescu: U.P.B. Sci. Bull., Series B, 73 (2011) 181-196.

[3] J. Macak, H. Tsuchiya, A. Ghicov, K. Yasuda, R. Hahan, S. Bauer, P. Schmuki: Curr Opin Solid State Mater Sci, 11 (2007) 3-18.

[4] D. Regonini, C. Bowen, A. Jaroenworaluck, R. Stevens: Mater Sci Eng, R, 74 (2013) 377-406. 
[5] V. Zwilling, M. Aucouturier, E. Darque-Ceretti: Electrochim Acta, 45 (1999) 921 929.

[6] S. Chatterjee, M. Ginzberg, B. Gersten, In: MRS Proceedings: Effect of anodization conditions on the synthesis of $\mathrm{TiO}_{2}$ nanopores, Cambridge Univ Press 2006, 0951E09-27.

[7] S. Bauer, S. Kleber, P. Schmuki: Electrochem Commun, 8 (2006) 1321-1325.

[8] L. Ren, Z. Ma, Y. Zhang, W. Liu, Z. Liao, K. Yang: J Mater Sci Tech, 30 (2014) 699-705.

[9] J. Liu, X. Zhang, H. Wang, K. Yang, L. Fangbing, L. Muqin, E. Zhang: Biomed Mater, 9 (2014) 12-20.

[10] D.W. Gong, C.A. Grimes, \& R.S. Singh: J Mater Res, 16 (2001) 3331-3334.

[11] H. Du, Q. Chen, R. C. Che, Z. Y. Yuan, L.M. Peng: Appl Phys Lett, 79 (2001) 3702.

[12] C. Qingyun, P. Maggie, K.V. Oomman, A.G. Craig: J Mater Res, 20 (2005) 230236.

[13] S. Seulgi, L. Kiyoung, S. Patrik Schmuki: J Am Chem Soc, 134 (2012) 1131611318.

[14] S. Berger, R. Hahn, P. Roy, P. Schmuki: Phys Status Solidi B, 247 (2010) 24242435.

[15] H. TsuchiyaJan, M. Macak, L. Taveira, E. Balaur, A. Ghicov, K. Sirotna, P. Schmuki: Electrochem Commun, 7 (2005) 576-580.

[16] J. M. Macak, T. Hiroaki Tsuchiya, S. Patrik Schmuki: Angew Chem, 44 (2005) 2100-2102.

[17] J. Liu, F. Li, C. Liu, H. Wang, B. Ren, K. Yang, E. Zhang: Mater Sci Eng C, 35, (2014) 392-400.

[18] E. Zhang, X. Wang, M. Chen, B. Hou: Mater Sci Eng C, 69 (2016) 1210-1221.

[19] H. Andrew, M. Amanda, H. Michael: Materials, 5 (10), (2012) 1890-1909.

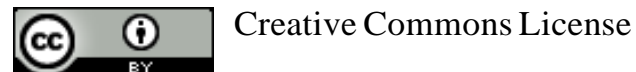

This work is licensed under a Creative Commons Attribution 4.0 International License. 